\title{
Psychoanalysis: Science or Nonscience?
}

Carola B. B. Mathers, Senior Registrar, Springfield Hospital and St George's Hospital, London SW17

The debate regarding the scientific status of psychoanalysis is considered by many psychiatrists to be a sterile one; this leads to a polarisation of views in which those in favour of psychoanalysis, feeling themselves to be losing, retreat to the position of stating that meaning is more important than scientific status, while those against argue that because psychoanalysis is unscientific it is meaningless and thus should not remain part of our psychiatric practice; the debate being reformulated as psychoanalysis: sense or nonsense? The consequence of giving up this debate is that it allows us also to give up the struggle to define science adequately to ourselves, and to question the relationship between psychiatry and science. Our understanding of science reflects directly on the quality of our research; this is particularly relevant to research in the practical application of psychoanalysis. Before we consider our understanding of science, however, we should consider our epistemological theories, or how we know things.

Empirical realism, one such theory, states that the only knowable things are those which we perceive through the senses, like this page of print; empirical because it deals with the objects of our sense-perceptions, and realist because it assumes a world 'out there'. A scepticist theory suspends judgements about certainty; we accept that some phenomena are real, but we cannot know which, since our sense-perceptions can and do deceive us, optical illusions being an example of such deception. Other theories state that it is possible to know certain things through their effects, if we cannot perceive them directly, for example a magnetic field which we can only perceive through its effects, but which we believe exists nevertheless.

According to our models of epistemology, we construct models of science. Traditional scientific activity, as exemplified by the physical sciences, corresponds to a realist theory, that science is the study of objects and/or their effects on the world, by means of experiments which test out our hypotheses and find them to be correct or incorroct. According to Popper ${ }^{1}$ science proceeds by imaginative hypotheses (conjectures) to observations designed to falsify these hypotheses (refutations). 'The criterion of the scientific status of a theory is its falsifiability, or refutability, or testability' (ibid.) We can see here that Popper implies that falsifiability is the same as testability; clearly they are not the same, since by testing a theory one can prove it to be correct or incorrect. If we agree with Popper's statement, that a discipline which cannot generate falsifiable theories cannot be a science, we exclude psychoanalysis without further thought.

However, there are several problems with Popper's philosophy of science. Firstly, scientists spend much of their time working on hypotheses, experimentally, proving them to be correct. Experiments are designed to produce observations which test the degree of truth in a hypothesis, they are not designed to prove it to be incorrect. Secondly, when a theory is challenged it is not rejected, as Popper suggests, but modified or subsumed under a theory which explains the phenomena more completely. For example, Newtonian theory has limited explanatory power and becomes incorrect beyond these limits, but it has been incorporated into Einsteinian theory, instead of being rejected. Thirdly, the problems of observations: it is well known that observations to a large extent depend on one's theories, so that one sees what one expects to see, and designs experiments which generate the data that one expects. (We are familiar with the methodological problems of unvalidated questionnaires). Popper attempts to solve this problem by assuming that proving our theories to be wrong will somehow purify our observations, but we still fall into the trap of expecting our data to show that we are wrong.

There is thus a fundamental discrepancy between what Popper says scientists do (a prescriptive model) and what they actually do (a descriptive model). While falsifability may be an ideal it does not characterise how scientists work, and thus cannot serve as an exclusion criterion to demarcate science from nonscience. I have laboured this point because it disturbs me to find some of my colleagues accepting Popper so uncritically and using his philosophy to justify their own positions.

A model which has recently attracted interest among psychiatrists and psychologists is 'transcendental realism' as expounded by Bhaskar ${ }^{2}$ and later Will. ${ }^{3.4}$ This theory states firstly that a world exists outside of men which transcends our human consciousness. Secondly, that science tries to understand the principles of this real world. Thirdly, that natural laws are real, not merely descriptions of patterns of events seen in experiments, and that they are descriptions of 'generative mechanisms' which cause observed patterns of events. Scientific activity develops in three stages: first, the empirical identification of a phenomenon using existing cognitive materials; second, a plausible explanation for the phenomenon is invented in the form of a possible generative mechanism; third, the reality of this imagined generative mechanism is tested empirically.

Science thus proceeds by moving from manifest phenomena to the structures that cause or generate them. Once the generative mechanism has been tested, it itself becomes the manifest phenomenon that is investigated by proposing a further generative mechanism. Science is therefore a stratified activity that corresponds to the real stratification of the phenomena studied. An example from chemistry illustrates this idea: a chemical reaction between an acid and a base is explained by a generative mechanism, 
namely the theory of atomic number and valency. This is in turn explained by a further generative mechanism, the theory of electrons and atomic structure. ${ }^{3}$

Phenomena in psychoanalysis are similarly stratified: an irrational emotional response by a patient towards the analyst is explained by the theory of transference, which is further explained by the theory of projection of unconscious material. In this way general psychoanalytic theories are constructed. They are tested in the 'laboratory' of psychoanalysis, the analytic session. For example, the analyst tests the validity of an interpretation by observing its effect on the patient. The response to a correct interpretation may vary: it may promote a stream of new material or it may be ridiculed or denied; an incorrect interpretation will have neither effect. Resistance to treatment is a generative mechanism thought to underlie phenomena such as denial of an accurate but threatening interpretation.

According to the transcendental realist model, then, different levels of scientific description correspond to different levels of reality; to attempt to reduce all scientific description to one level is to deny that reality has different levels. It would clearly be inappropriate to attempt to explain all reality in terms of neurochemistry, or sociology, or psychoanalysis. A useful research task would therefore be, as Will suggests, to draw up a typology of scientific theories which would define the boundary of each field of enquiry. This would avoid the reductionism often found in pseudoscientific circles in which one explanation is sought for all observed phenomena, for example the attempt to explain an individual's emotional life exclusively in terms of neuroanatomy, systems theory or whichever theory is enjoing temporary popularity.

Unfortunately the transcendental realist theory avoids the problem of objectivity, which a realist theory must consider, since it purports to describe real phenomena. In the framework of psychoanalysis the problem of describing real phenomena is evident: one cannot 'see' transference or resistance, and a sceptic (in the lay sense) might argue that he could not perceive these phenomena through their effects either. This problem of the independence of the observer from the phenomena is an equally pressing one in the field of subatomic physics: experiments have shown that the observer significantly influences the observed; in the realm of subatomic phenomena there is no such thing as a real world independent of us as observers, so that we inevitably become participants in our experiments. This is an example of the stratification of levels of explanation: Newtonian physics and everyday life tell us that a table is a table and remains so whether we are in the room or not; however quantum mechanics tells us that we influence particles in the table merely by our presence.

Experimenters therefore have to include themselves in their experiments; this is causing enormous difficulties in physies laboratories, as one may imagine. The position of the analyst now looks remarkably similar to that of the physicist: the analyst recognises that he or she influences the patient and takes this into account, in fact uses his or her own reactions to the patient as valuable data. On the basis of the data from the analytic session and using his or her knowledge of psychoanalytic theory the analyst makes predictions about the patient and tests these out in subsequent sessions. The physicist too cannot make any predictions with certainty; he or she deals in probabilities only.

My own opinion is that as psychiatrists we need to keep open minds as to what constitutes scientific activity, and to question constantly our own hypotheses; for, to consider levels of explanation unfamiliar to us as being nonscience (or nonsense) is to impoverish our understanding, and thus our practice.

\section{Rererences}

${ }^{1}$ POPPER, K. R. (1957) Philosophy of science: a personal report British Philosophy in Mid-Century, (ed. C. A. Mace), London: Allen \& Unwin.

${ }^{2}$ BHASKAR, R. (1975) A Realist Theory of Science 2nd. $\infty$ d. Brighton: Harvester Press.

${ }^{3}$ WILL, D. (1980) Psychoanalysis as a human science. British Journal of Medical Psychology. 53, 201-211.

4 - (1983) Transcendental realism and the scientificity of psychoanalysis: a reply to recent criticism. British Journal of Medical Psychology, 56, 371-378.

\section{Royal Academy Soirée}

Members are reminded that on Thursday, 7 August 1986 the second joint Medical Royal Colleges and Faculties Soire will take place at the Royal Academy, Burlington House during the Royal Academy's 1986 Summer Exhibition. It will take place between 6.45 p.m. and 8.45 p.m. and dress will be informal. The ticket price is $£ 16$ per head, which includes access to the Academy's Summer Exhibition, buffet supper and wine. Tickets must be obtained, not later than 22 July 1986, from the Secretary of this College. Cheques should be made payable to the Royal College of Psychiatrists.

\section{Child and Adolescent Psychiatry}

The College's Child and Adolescent Psychiatry Specialist Section is now inviting submissions for a session of ten-minute presentations by senior registrars in child and adolescent psychiatry to be held as part of the Sheffield Residential Conference from 11 to 13 September 1986. Those interested in submitting papers should contact Dr Ian Berg, Department of Psychological Medicine (Children), The Clarendon Wing, The General Infirmary at Leeds, Belmont Grove, Leeds LS2 9NS, as soon as possible. 